\title{
Dynamical overlap fermions in the epsilon-regime
}

\author{
JLQCD collaboration: Hidenori Fukaya ${ }^{* a}$, Shoji Hashimoto, ${ }^{b c}$ Kazuyuki Kanaya, ${ }^{d}$ \\ Takashi Kaneko, ${ }^{b c}$ Hideo Matsufuru ${ }^{b}$ Kenji Ogawa, ${ }^{e}$ Masataka Okamoto, ${ }^{b}$ \\ Tetsuya Onogi, ${ }^{f}$ Norikazu Yamada. ${ }^{b c}$ \\ ${ }^{a}$ Theoretical Physics Laboratory, RIKEN, Wako 351-0198, Japan \\ ${ }^{b}$ High Energy Accelerator Research Organization (KEK), Tsukuba 305-0801, Japan. \\ ${ }^{c}$ School of High Energy Accelerator Science, The Graduate University for Advanced Studies \\ (Sokendai), Tsukuba 305-0801, Japan. \\ ${ }^{d}$ Graduate School of Pure and Applied Sciences, University of Tsukuba, Tsukuba 305-8571, \\ Japan \\ ${ }^{e}$ Department of Physics, National Taiwan University, Taipei 10617, Taiwan. \\ ${ }^{f}$ Yukawa Institute for Theoretical Physics, Kyoto University, Kyoto 606-8502, Japan.
}

\begin{abstract}
We report on the two-flavor QCD simulation in the $\varepsilon$-regime using the overlap fermion formulation. Sea quark mass is reduced to $\sim 2 \mathrm{MeV}$ on a $16^{3} \times 32$ lattice with the lattice spacing $a$ $\simeq 0.11 \mathrm{fm}$. Topological charge is fixed at $Q=0$. We compare the eigenvalue distribution of the overlap-Dirac operator with the prediction of the chiral random matrix theory. Preliminary results on meson correlators are also reported.
\end{abstract}

XXIVth International Symposium on Lattice Field Theory

July 23-28, 2006

Tucson, Arizona, USA

\footnotetext{
* Speaker.

†E-mail: hfukaya@riken.jp
} 


\section{Introduction}

In the standard lattice QCD simulations, large volume, chiral, and continuum limits have to be approached in order to obtain reliable physical quantities. That is very challenging even with the fastest supercomputers currently available. In most of the previous works, the chiral limit was sacrificed and the simulations have been done with considerably heavier up and down quarks, with non-chirally symmetric Dirac operators, such as the Wilson or KS-type fermions.

Recently, an alternative approach, which gives a priority to the chiral limit rather than the infinite volume, is pursued based on the understanding of the finite volume effect using chiral effective theory. That is the lattice simulation in the $\varepsilon$-regime. For such a simulation, the exact chiral symmetry for the lattice fermion is essential as one treats extremely small quark masses of $O(1 \mathrm{MeV})$. We use the overlap Dirac operator [1]

$$
D=m_{0}\left(1+\gamma_{5} \operatorname{sgn} H_{W}\right)
$$

which has the exact chiral symmetry through the Ginsparg-Wilson relation [2]. $H_{W}=\gamma_{5} D_{W}\left(-m_{0}\right)$ is the standard hermitian Wilson-Dirac operator with large negative mass $-m_{0}$.

If the size of the box, $L$, is small and satisfies

$$
1 / \Lambda_{Q C D} \ll L \ll 1 / m_{\pi},
$$

where $\Lambda_{Q C D}$ the QCD scale and $m_{\pi}$ the pion mass, an expansion in terms of $\varepsilon^{2}=m_{\pi} / \Lambda_{U V}\left(\Lambda_{U V}\right.$ is an UV cutoff, e.g. $4 \pi F_{\pi}$ ) is valid after a careful treatment of the zero-momentum modes [3, 4]. In such a small physical volume, the $\varepsilon$-regime, the low-energy constants, such as the chiral condensate $\Sigma$ and the pion decay constant $F_{\pi}$, can be extracted through the current correlators. Since the quark mass is already very small, the chiral extrapolation is not necessary.

Most previous lattice simulations in the $\varepsilon$-regime were limited to the quenched approximation [5] except for a few pioneering works with rather heavier quarks [6]. The new project by the JLQCD collaboration started in March 2006 is aiming at performing large scale simulations of QCD with the overlap fermion formulations. We also explore the $\varepsilon$-regime by pushing the quark mass down to a few $\mathrm{MeV}$. We employ topology conserving actions to assure the overlap fermion determinant to be smooth $[8]$.

For the gauge action we use the Iwasaki action with $\beta=2.30-2.35$. With many additional algorithmic efforts [9] we have generated thousands of configurations of two-flavor QCD on a $16^{3} \times 32$ lattice. The lattice spacing is $a \sim 0.11-0.125 \mathrm{fm}$. As an exploratory run we attempted to reduce the quark mass down to $2 \mathrm{MeV}$ at $\beta=2.35$, at which $L \sim 1.8 \mathrm{fm}$. With this small quark mass the condition (1.2) is safely satisfied.

In this report we concentrate on the study with the smallest sea quark mass $m a=0.002$ ( $m \sim$ $2 \mathrm{MeV}$ ) at $Q=0$. We first report on the numerical cost for this simulation in Sec. 2, and describe the determination of the lattice spacing in Sec. 3. Preliminary results for the low-energy constants, $\Sigma$ and $F_{\pi}$, are obtained through the low-lying eigenvalues (Sec. 44) and through the pion correlators (Sec. 5). The summary and discussion are given in Sec.6. 




Figure 1: The history of CG iteration along the HMC steps. The sea quark mass dependence seems weak when $m a \lesssim 0.03$, and the numerical cost with $m a=0.002$ (red) is almost the same as the case with $m a=0.02$ (blue).

\section{Numerical cost}

Naively, the simulation cost increases for small quark masses as an inverse power of the quark mass $m$, and tends to diverge near the massless limit. It is, however, not the case in the finite volume. The numerical cost or the iteration count for the solver, say the conjugate gradient (CG) solver, is roughly proportional to the condition number

$$
\left|\lambda_{\max }+m\right|^{2} /\left|\lambda_{\min }+m\right|^{2},
$$

where $\lambda_{\min , \max }$ denotes the lowest (highest) eigenvalue of the overlap Dirac-operator. In the largevolume limit, the numerical cost is primarily determined by the quark mass since $\lambda_{\min } \sim 1 / \Sigma V \sim 0$ and $\lambda_{\max }$ is insensitive to the gauge configuration. In the small quark mass limit at a fixed $V$, on the other hand, $m$ is made smaller than $1 / \Sigma V$ and $\left\langle\lambda_{\min }\right\rangle \simeq z / \Sigma V$ determines the condition number (2.1) ${ }^{1}$. Here, the numerical factor $z$ can be estimated using the chiral random matrix theory. For $N_{f}=2$ and $Q=0$, it is $z \simeq 4.34$.

In fact, as Fig. 1 1 shows, the quark mass dependence of the iteration count is weak for $a m \lesssim$ 0.03 , and the simulation cost for $a m=0.002$, which corresponds to $m \sim 2 \mathrm{MeV}$ (with an assumption for the renormalization factor $Z_{m}=1.8$ as in the quenched theory) is almost the same as that for ten times heavier quark mass $a m=0.020$. However, we should note that the auto-correlation time seems longer for smaller quark mass. We do not have enough statistics to precisely calculate, but we expect it is $O(100)$ trajectories.

On a half-rack (512 nodes) of the IBM BlueGene/L (2.8 Tflops peak performance), we need roughly one hour per trajectory. In the following analysis, we use 100 configurations in $Q=0$ topological sector sampled from 1400 trajectories (first 400 are discarded for the thermalization). For the details of our numerical simulations, we refer to the contribution by Matsufuru [9].

\footnotetext{
${ }^{1}$ When there are zero modes, $\lambda_{\min }=0$, one must explicitly subtract them from the determinant.
} 

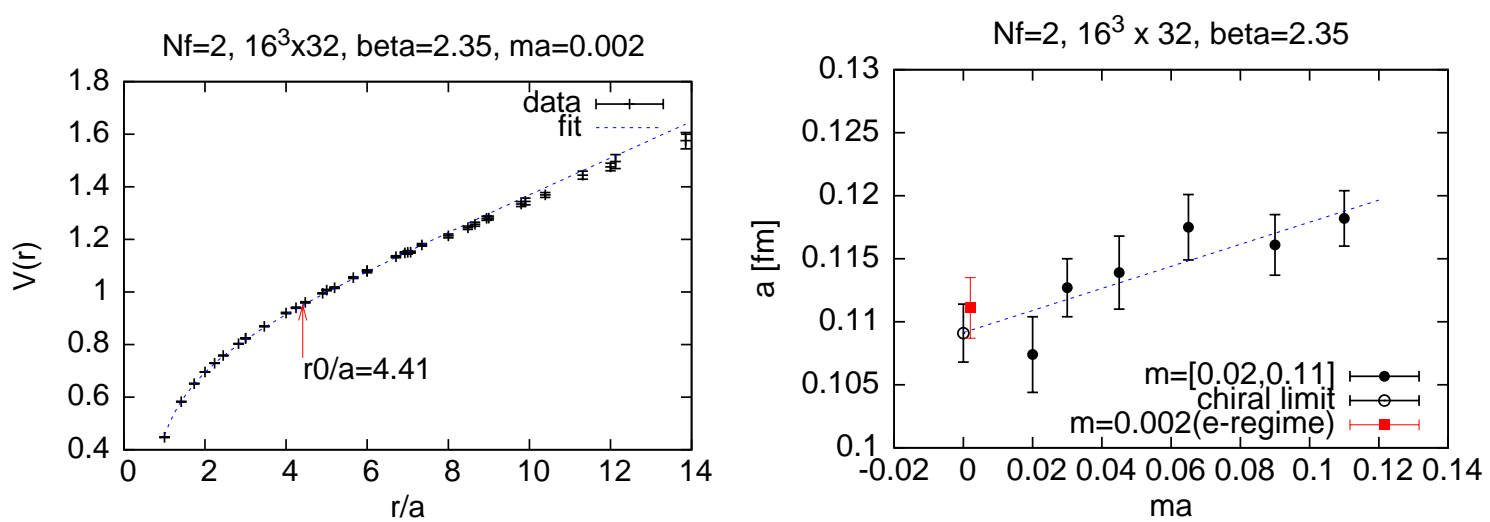

Figure 2: The static quark potential (left) and the lattice spacing with different quark mass (right). The quark potential has no indication of string breaking and $a \sim 0.11$ can be obtained, which is also consistent with the chiral extrapolation from the heavier quark mass points, as the dotted line in the right panel shows.

\section{Static quark potential with almost massless sea quarks}

To determine the lattice spacing $a$ we measure the static quark potential. In a very small quark mass regime one might expect the string breaking, but our results in Fig. 2 (left panel) show no indication. This is probably because the volume is too small to contain two static-light mesons. The overlap of the Wilson loop with the two static-light states could also be a problem.

We calculate the Sommer scale $r_{0}$ as usual and obtain $a \sim 0.111(2)$ fm (with an input $r_{0}=$ $0.49 \mathrm{fm}$ ), which is consistent with the chiral extrapolation from the heavier quark mass points as shown in the right panel.

\section{Low eigenmodes}

We expect that the eigenvalue distribution of the Dirac operator is well described by the chiral random matrix theory (ChRMT) in the $\varepsilon$-regime. We compare them with our lattice data.

The eigenvalues of the overlap-Dirac operator lie on a circle in the complex plane, and we project them as

$$
\lambda_{i} \equiv \frac{\operatorname{Im} \lambda_{i}^{o v}}{1-\operatorname{Re} \lambda_{i}^{o v} / 2}
$$

where $\lambda_{i}^{o v}$ is the $i$-th complex eigenvalues of the overlap-Dirac operator $D$. Note that $\lambda_{i}$ is very close to $\operatorname{Im} \lambda_{i}^{o v}$ for the low-lying modes.

ChRMT predicts that the lowest mode with an eigenvalue $\lambda_{1}$ in $N_{f}=2$ theory feels strong repulsive force from zero. That is more prominent than the quenched case, and numerically one obtains [10]

$$
\left\langle\lambda_{1}\right\rangle \Sigma V=1.77\left(N_{f}=0\right), \quad\left\langle\lambda_{1}\right\rangle \Sigma V=4.34\left(N_{f}=2\right),
$$

where the chiral condensate $\Sigma$ could depend on the number of flavors. The HMC history of $\lambda_{1}$ shown in Fig. 3 is consistent with this theoretical expectation. The lowest eigenvalue is pushed up more for lighter quarks than for heavier quarks. 




Figure 3: The HMC history of the lowest eigenvalue of $D$ calculated every 10 trajectories.
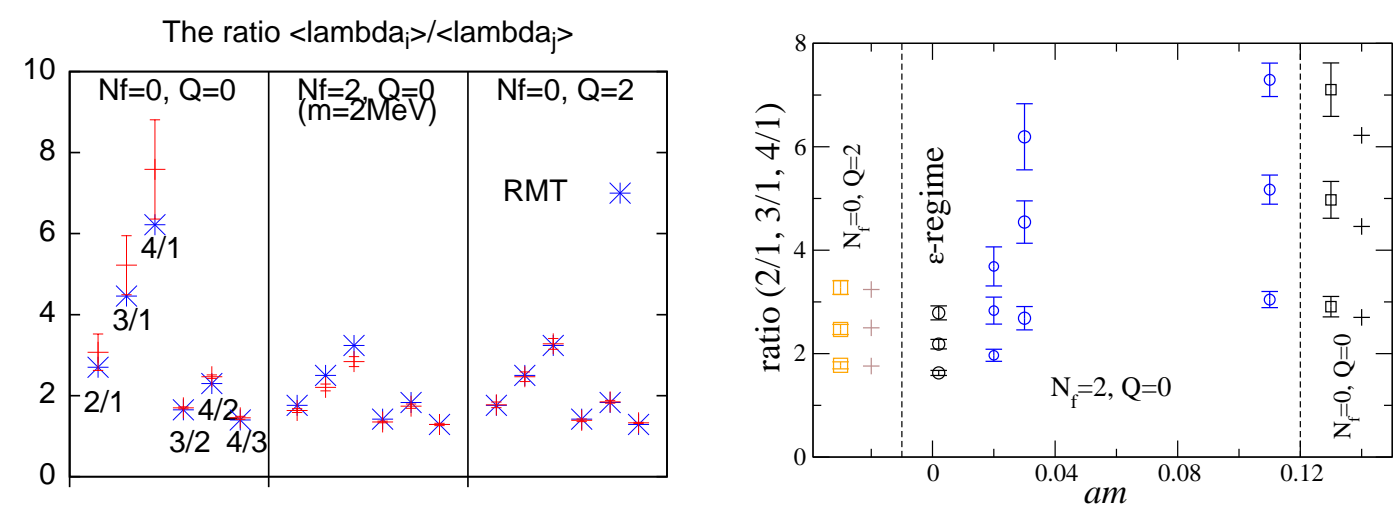

Figure 4: Ratio of the low-lying eigenvalues. " $i / j$ ” denotes $\lambda_{i} / \lambda_{j}$. Left: we plot the data with $\left(N_{f}, Q\right)=$ $(0,0),(2,0),(0,2)$ (red crosses). The ChRMT predictions are shown by blue stars. Right: quark mass dependence of the ratios " $2 / 1$ ", " $3 / 1$ " and " $4 / 1$ ". Crosses are ChRMT results with $\left(N_{f}, Q\right)=(2,0)=(0,2)$ on the left, and $\left(N_{f}, Q\right)=(0,0)$ on the right, where we also plot the corresponding quenched results (squares). Circles are lattice data in two-flavor QCD.

Since the value of chiral condensate cannot be directly compared for different sea quark masses, it is convenient to look at the dimensionless ratio of the eigenvalues $\lambda_{i} / \lambda_{j}$. We present some of such ratios of low-lying eigenvalues on the left panel of Fig. 4 In the plot, the results are shown for both quenched and unquenched $\left(N_{f}=2\right)$ cases at $Q=0$, as well as for the quenched data at $Q=2$. All the data show a good agreement with ChRMT (blue star symbols). ChRMT predicts that the $\left(N_{f}, Q\right)=(2,0)$ theory has the same low-lying eigenvalue spectrum as the $\left(N_{f}, Q\right)=(0,2)$ theory, which is well reproduced by the lattice data.

It is also interesting to see the quark mass dependence of the ratios (the right panel of Fig. (4). The data with $a m \geq 0.03$ are close to the quenched ChRMT, which is shown by the crosses on the right end, and below $a m=0.03$ they suddenly drop to the $N_{f}=2$ results (crosses on the left end).

We obtain the lattice bare value of $\left(\Sigma^{N_{f}=2}\right)^{1 / 3}=228.9(3.6) \mathrm{MeV}$ from $\lambda_{1}$ measured in am $=$ 0.002 configurations. Here, the renormalization of $\Sigma$ is not taken into account. 

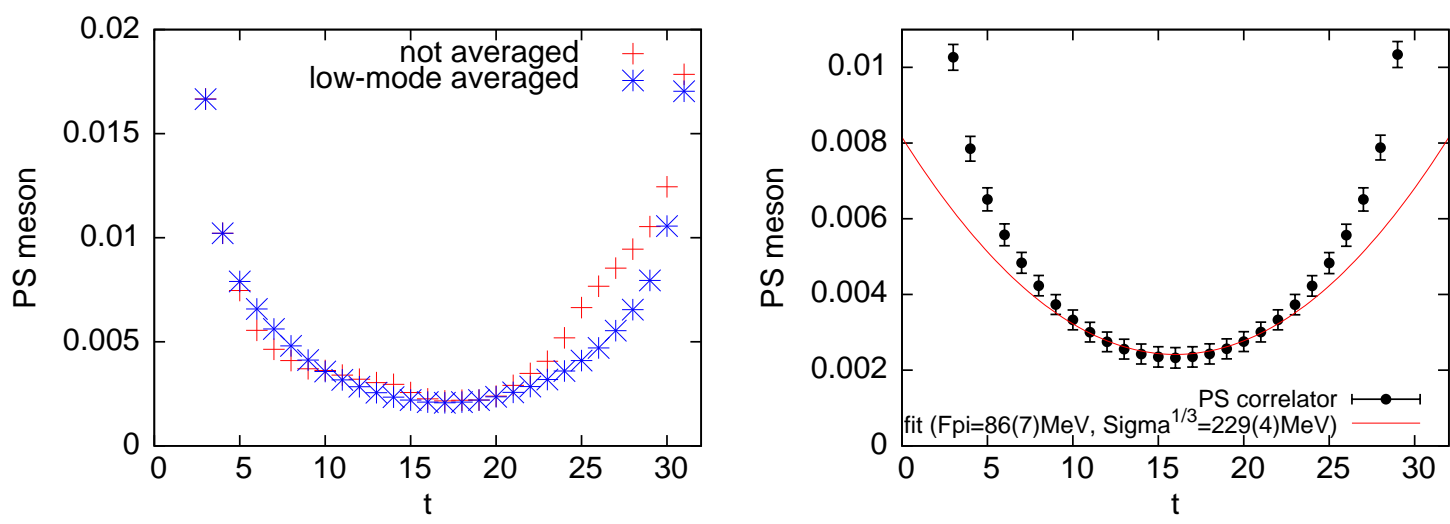

Figure 5: The left panel shows the effect of low-mode averaging (LMA) on the pion propagator(for one configuration): data with (blue stars) and without (red crosses) LMA are shown for comparison. A quadratic fit is attempted for the LMA pion propagator on the right panel.

\section{Pion correlators in the $\varepsilon$-regime}

The pion correlators in the $\varepsilon$-regime are largely deformed by the finite volume effects, and no longer an exponential but a quadratic function of time $t$,

$$
\begin{aligned}
& \int d^{3} x\left\langle P^{a}(x, t) P^{b}(0,0)\right\rangle^{Q}=\delta^{a, b}\left[A(t / T-1 / 2)^{2}+B\right], \\
A= & \frac{T \Sigma^{2}}{F_{\pi}^{2}}\left[\left(\frac{\Sigma_{Q}(\mu)}{\Sigma}\right)^{2}+\frac{\Sigma^{\prime}(\mu)}{2 \Sigma}+\frac{\Sigma_{Q}(\mu)}{\mu \Sigma}\right], B=\frac{\Sigma_{Q}\left(\mu^{\prime}\right)}{m T},
\end{aligned}
$$

where $\mu=m \Sigma V$ and $\mu^{\prime}=\mu\left(1+3 \beta_{1} / 2 F_{\pi}^{2} V^{1 / 2}\right)$. The effective chiral condensate $\Sigma_{Q}(\mu)$ has strong dependences on $Q$ and $m$, which are expressed by the modified Bessel functions (see [4] for the details). Here, $\langle O\rangle^{Q}$ denotes the expectation value in the $Q$ topological sector.

We calculate the pion correlators on our lattice using the low-mode averaging (LMA) technique [11]. We find that $\sim 90 \%$ contribution comes from the lowest 100 modes of the Dirac operator, and the LMA is very effective, as the left panel of Fig. 5 shows.

With an input $\Sigma^{1 / 3}=229$ (4) MeV from $\lambda_{1}$, the quadratic fit to the correlator works well as shown on the right panel. We obtain a preliminary result $F_{\pi}=86(7) \mathrm{MeV}$ with a fitting range $t=[10,22]$, for which $\chi^{2} /$ dof $\simeq 0.25$. The result is very preliminary, not only because the statistics is limited so far but detailed analysis of the systematic errors is yet to be performed.

\section{Summary}

We pushed the dynamical overlap fermion simulation towards the chiral limit and in fact succeeded a simulation at $m \sim 2 \mathrm{MeV}$ on the lattice with $L \sim 1.8 \mathrm{fm}$ and $a \sim 0.11 \mathrm{fm}$. On the finite volume lattice, the $\mathrm{CG}$ count does not diverge in the chiral limit as expected from the chiral effective theory. The distribution of low-lying eigenvalues of $D$ is consistent with the ChRMT expectation, from which we may extract the value of the chiral condensate. 
The measurement can be extended to the hadron correlators. As a first test, we calculate the pion correlator and find that the LMA works extremely well in the $\varepsilon$-regime. Precise determination of $F_{\pi}$ is feasible with such calculations.

So far, our simulation has been done at a single parameter set, $Q=0$ and $a m=0.002$. To study other topological sectors and different quark masses is very important, because in the $\varepsilon$-regime $Q$ and $m$ dependences are prominent and help to improve the sensitivity to the low-energy constants.

Numerical studies are performed on Hitachi SR11000 and IBM Blue Gene at High Energy Accelerator Research Organization (KEK) under a support of its Large Scale Simulation Program (No. 06-13). Some parts of numerical analysis were carried out on SX8 at YITP in Kyoto University. This work is supported in part by the Grant-in-Aid of the Ministry of Education (No. 13135213, 16740156, 17340066, 17740171, 18034011, 18340075, 18740167).

\section{References}

[1] H. Neuberger, Phys. Lett. B 417, 141 (1998), H. Neuberger, Phys. Lett. B 427, 353 (1998).

[2] P. H. Ginsparg and K. G. Wilson, Phys. Rev. D 25, 2649 (1982).

[3] F. C. Hansen, Nucl. Phys. B 345, 685 (1990), F. C. Hansen and H. Leutwyler, Nucl. Phys. B 350, 201 (1991), H. Leutwyler and A. Smilga, Phys. Rev. D 46, 5607 (1992),

[4] P. H. Damgaard, M. C. Diamantini, P. Hernandez and K. Jansen, Nucl. Phys. B 629, 445 (2002),

[5] L. Giusti, M. Luscher, P. Weisz and H. Wittig, JHEP 0311, 023 (2003), K. Ogawa and S. Hashimoto, Prog. Theor. Phys. 114, 609 (2005), H. Fukaya, S. Hashimoto and K. Ogawa, Prog. Theor. Phys. 114 (2005) 451, P. Hernandez, et al, these proceedings.

[6] T. A. DeGrand and S. Schaefer, Phys. Rev. D 72, 054503 (2005), P. H. Damgaard, U. M. Heller, K. Splittorff, B. Svetitsky and D. Toublan, arXiv:hep-lat/0602030, T. DeGrand, R. Hoffmann, S. Schaefer and Z. Liu, Phys. Rev. D 74, 054501 (2006), T. DeGrand, Z. Liu and S. Schaefer, arXiv:hep-lat/0608019, J. Bloch and T. Wettig, arXiv:hep-lat/0609020, K. Splittorff et al, these proceedings, N. Cundy et al, these proceedings, A. Hart et al, these proceedings.

[7] S. Schaefer, arXiv:hep-lat/0609063.

[8] P. M. Vranas, arXiv:hep-lat/0001006, H. Fukaya, S. Hashimoto, K. I. Ishikawa, T. Kaneko, H. Matsufuru, T. Onogi and N. Yamada [JLQCD Collaboration], arXiv:hep-lat/0607020,

S. Hashimoto et al., PoS(LAT2006)052

[9] T. Kaneko et al., PoS(LAT2006)054, H. Matsufuru et al., PoS(LAT2006)031, N. Yamada et al., PoS(LAT2006)060.

[10] P. H. Damgaard and S. M. Nishigaki, Phys. Rev. D 63, 045012 (2001).

[11] T. DeGrand and U. M. Heller [MILC collaboration], Phys. Rev. D 65, 114501 (2002),

L. Giusti, P. Hernandez, M. Laine, P. Weisz and H. Wittig, JHEP 0404, 013 (2004). 